\section{P-TEFb et Brd4}

Acteurs de la levée de pause transcriptionnelle, possibles cibles thérapeutiques

Alessandro Furlan, Florence Agbazahou, Mélanie Henry, Mariano Gonzalez-Pisfil, Corentin Le Nézet, Dorian Champelovier, Marie Fournier, Bernard Vandenbunder, Gabriel Bidaux, Laurent Héliot

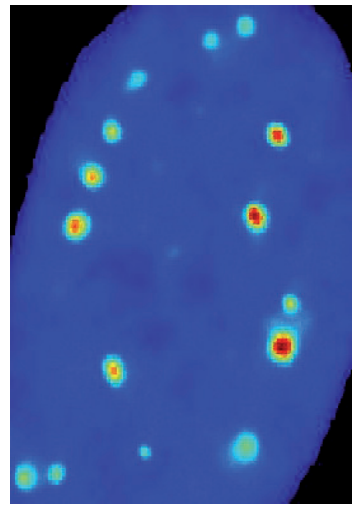

CNRS UMR 8523, Laboratoire de physique des lasers, atomes et molécules, université de Lille, 59655 Villeneuve d'Ascq, France alessandro.furlan@univ-lillel.fr
En fonction des conditions rencontrées dans son microenvironnement, la cellule adapte ses programmes transcriptionnels pour assurer son intégrité et celle de l'organisme. Les mécanismes les plus fréquemment décrits pour réguler la transcription font intervenir la fixation de combinaisons de facteurs de transcription spécifiques sur les promoteurs des gènes concernés. Les cellules ont cependant mis en place de nombreux autres niveaux de régulation. Parmi ceux-ci figurent notamment les séquences régulatrices de type enhancers, les mécanismes d'épissage intervenant en parallèle de l'élongation des transcrits, ou encore les modifications épigénétiques affectant l'état de la chromatine. Dans les cellules eucaryotes, l'initiation de la transcription est suivie d'une mise en pause, une cinquantaine de nucléotides en aval du site d'initiation, pour environ $60 \%$ des gènes [1]. Cette revue se focalise sur l'intérêt thérapeutique potentiel des acteurs moléculaires impliqués dans la levée de cette pause.

\section{Quelques acteurs importants: la polymérase, le complexe PTEF-b et leurs partenaires}

L'ARN polymérase II (ARN pol II), responsable de la transcription des ARN messagers (ARNm) des cellules euca-

Vignette (Photo ๑ Alessandro Furlan) ryotes, est un complexe supramoléculaire constitué de 12 sous-unités dont la masse moléculaire globale dépasse les 500 kDa. La sous-unité porteuse de l'activité polymérase, Rpbl, est une protéine d'environ 250 kDa qui possède à son extrémité C-terminale (CTD pour C-terminal domain) une répétition de 52 hexapeptides YSPTSPS ${ }^{1}$ cruciaux pour la régulation de l'élongation de la transcription [2]. La polymérase s'arrête en effet généralement de transcrire une cinquantaine de nucléotides en aval du site d'initiation de la transcription, sous l'effet des facteurs associés DSIF (DRB sensitivity inducing factor) et NELF (negative elongation factor). Ce complexe de pause est désactivé par la phosphorylation au niveau des sérines en position 2 (Ser2) du CTD de la polymérase et des protéines DSIF et NELF.

Le complexe P-TEFb (positive transcription elongation factor $b$ ) est porteur de l'activité kinase capable d'achever cette pause [3]. P-T\&Fb est un dimère constitué de la kinase cdk9 (cyclin-dependent kinase 9) et de la cycline $\mathrm{Tl}$ (ou T2, plus rarement). La cycline Tl (CTl) module l'activité de cdk9 et joue un rôle d'intégrateur des signaux cellulaires. Elle possède ainsi différents domaines d'interactions avec diverses protéines, dont I'ARN pol II, HEXIMI (hexamethylene bisacetamide inducible 1), mais aussi l'ARN non codant 7SK (Figure 1) (pour une revue, voir [4]).

P-TEFb peut se complexer avec I'ARN 7SK et les protéines HEXIMI, LARP7 (La-related protein 7) et MePCE (methyl capping enzyme), qui constituent la ribonucléoprotéine (RNP) 7SK $(\rightarrow$ ) Voir la Synthèse (pour revue, voir [5]) $(\rightarrow$ ). 


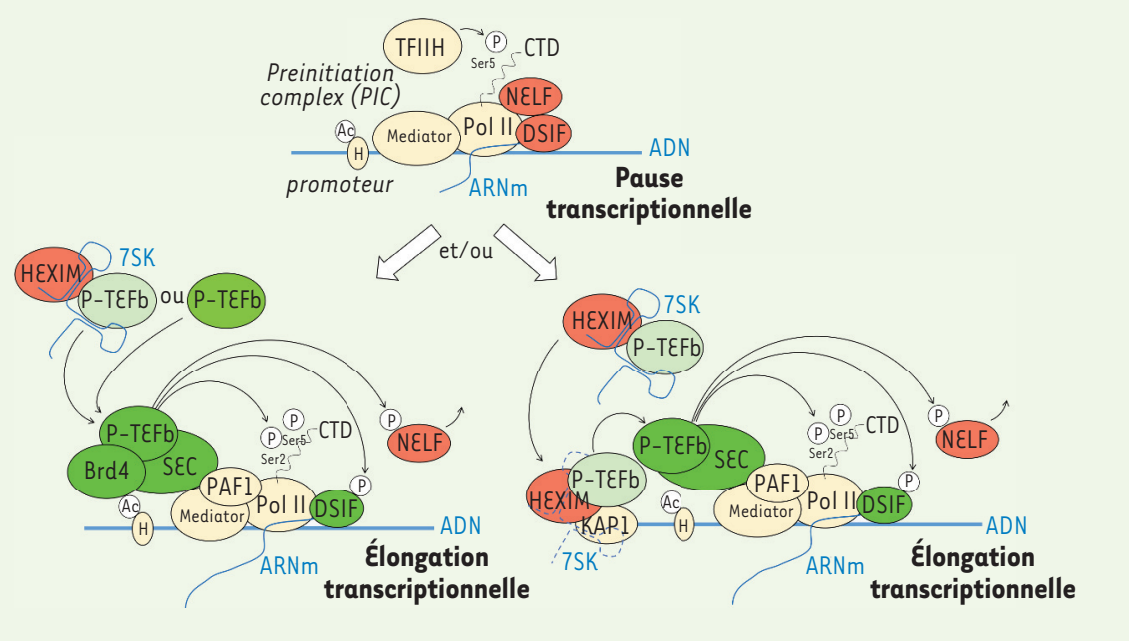

Figure 1. Les principaux acteurs connus des mécanismes de levée de pause transcriptionnelle. L'activité de I'ARN polymérase II (Pol II) est précédée par la formation du PIC (pre-initiation complex) au niveau du promoteur du gène. Le PIC est composé des facteurs généraux de transcription, TFII A, B, D, $\varepsilon$ et $H$, interagissant avec le complexe Mediator et Pol II. TFII H phosphoryle le CTD (C-terminal domain) de Pol II sur les résidus Ser5 et permet l'initiation de la transcription. Pol II entre ensuite en pause, une cinquantaine de nucléotides en aval du site d'initiation de la transcription pour la majorité des gènes, sous

l'action notamment des facteurs négatifs d'élongation DSIF (DRB sensitivity inducing factor) et NELF (negative elongation factor). La levée de pause est assurée principalement par P-TEFb (positive transcription elongation factor), qui va phosphoryler ( $P$ » encerclés sur la figure) la Ser2 du CTD, ainsi que DSIF et NELF. DSIF reste associé à la polymérase et se transforme en facteur positif d'élongation, tandis que NELF se dissocie de la polymérase qui commence l'élongation productive. P-TEFb existe sous forme libre ou sous une forme inactive complexée avec HEXIM (hexamethylene bisacetamide inducible 1) et l'ARN 7SK entre autres au sein de la ribonucléoprotéine 7SK (RNP 7SK). P-TEFb peut être recruté de différentes manières aux sites de transcription. Pour des soucis de clarté du schéma, nous avons représenté côte à côte deux modes de recrutement, bien que ceux-ci ne soient pas les seuls possibles et non exclusifs. La protéine Brd4 (bromodomain-containing protein 4), qui se fixe au niveau des histones acétylées (forme ovale notée « $H$ », avec «Ac » encerclé sur la figure) peut notamment induire la dissociation de P-TEFb d'HEXIM et 7SK et permettre son recrutement. Le complexe Mediator et PAFl (polymerase-associated factor 1) participent aussi au recrutement de P-TEFb, associés au complexe multimérique SEC (super elongation complex). En parallèle, $\mathrm{P}$-TEFb peut également être recruté au niveau des promoteurs sous sa forme complexée avec la RNP 7SK, via l'interaction de cette dernière avec KAPI (KRAB-interacting protein 1). La dégradation de l'ARN 7SK permet alors la libération in situ de P-TEFb actif.

L'activité kinasique de P-TEFb est inhibée au sein de ce complexe $[6,7]$. Un équilibre se forme dans les cellules, entre les formes libres de P-TEFb et celles associées à la RNP 7SK. Plusieurs protéines sont capables de dissocier $\mathrm{P}-\mathrm{T}$-TFb de ce super-complexe, particulièrement en conditions de stress, et ainsi de favoriser la levée de pause et l'élongation transcriptionnelle. P-TEFb peut, notamment, être libéré par différents signaux de transduction dont Akt (protéine kinase B) et ERK (extracellular signal-regulated kinase) (pour revue, voir [8]).

\section{Plusieurs scénarios possibles pour la levée de pause}

Nous proposons dans la Figure 1, une synthèse des acteurs majeurs impliqués dans la levée de pause transcriptionnelle au regard des dernières avancées sur le sujet.

La protéine Brd4 (bromodomain-containing protein 4) appartient à la famille de protéines BET (bromodomain and extraterminal domain) qui reconnaissent les histones acétylées. Les premiers travaux sur Brd4 et la levée de pause suggéraient le recrutement de P-TEFb sur les promoteurs sous sa forme active, non associée à la ribonucléoprotéine 7SK [9]. Des travaux récents mettent cependant en évidence au niveau des promoteurs un ancrage de la RNP 7SK via KAPl (KRAB interacting protein 1) qui favorise ainsi le recrutement de $P$-TEFb [10]. CTl possède un domaine d'interaction avec l'ARN pol II qui pourrait également participer au recrutement de P-T\&Fb. Nous avons toutefois montré, par des approches de microscopie de type $\mathrm{FRET}^{2}$ en cellules vivantes, que la perte d'interaction entre P-TEFb et I'ARN pol II, suite à la mutation de ce domaine, ne perturbe pas la localisation de P-TEFb à proximité de la chromatine [11].

Au-delà du recrutement de P-TEFb, Brd4 peut aussi agir sur la levée de pause, en recrutant au niveau d'enhancers, la déméthylase JMJD6 (Jumonji C-domain-containing protein 6) qui élimine des marques de répression de la chromatine (méthylation symétrique de l'histone $\mathrm{H} 4$ sur le résidu argininine en position 3, H4R3me2s) et déstabilise I'ARN 7SK [12]. Brd4 possède également une activité HAT (histone acetyltransferase) [13]. Elle aurait, de surcroît, un rôle dans le contrôle des stress de torsion de l'ADN après la levée de pause [14]. Ainsi, Brd4 a été présenté comme un acteur clé de la levée de

${ }^{2}$ Transfert d'énergie entre molécules fluorescentes : il s'agit d'un transfert d'énergie entre deux molécules lorsqu'elles sont adjacentes, ce qui permet d'évaluer leur interaction. 


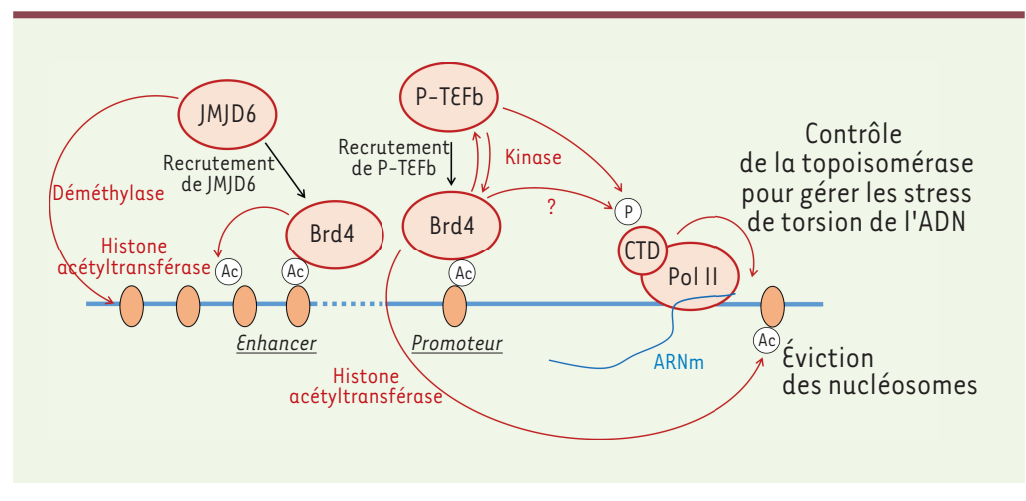

Figure 2. Brd4, une protéine aux fonctions multiples dans la transcription. Brd4 (bromodomain-containing protein 4) est une protéine ayant une activité pléiotropique possédant notamment des activités kinase et histone acétyltransférase (HAT). Via ses bromodomaines, Brd4 interagit avec les histones acétylées (Ac). Son activité HAT lui permet d'induire le décompactage de la chromatine et l'éviction des nucléosomes, nécessaires à la transcription. Cette activité HAT lui confère également un rôle dans le maintien des marquages épigénétiques lors de la mitose. Brd4 est une kinase atypique (l'activité kinase est portée

par plusieurs motifs séparés dans la séquence primaire de la protéine) potentiellement capable de phosphoryler P-TEFb (positive transcription elongation factor) et le CTD (domaine C-terminal) de l'ARN polymérase II («P » encerclé sur la figure) - cette activité reste sujette à controverse. Outre le recrutement de P-TEFb, Brd4 recrute la déméthylase JMJD6 (Jumonji C-domain-containing protein 6) au niveau d'enhancers appelés antipause enhancers, où JMJD6 retire des marques de répression de la transcription. Enfin Brd4 contribue à l'activation complète de la topoisomérase 1 pour ajuster les stress de torsion de l'ADN lors de l'élongation transcriptionnelle.

pause (Figure 2), pouvant même phosphoryler le CTD de la polymérase sur les résidus Ser2, en court-circuitant cdk9. Toutefois, Brd4 est une kinase atypique : elle ne possède pas de motif kinase consensus, mais plusieurs motifs similaires dispersés, et son activité de phosphorylation directe du CTD est controversée. De plus, l'existence d'un dialogue entre les kinases $c d k 7, c d k 9$ et Brd4 suggère qu'un réseau de rétrocontrôles module la phosphorylation du CTD de l'ARN pol II par ces kinases [15], ce qui va à l'encontre du concept de Brd4 agissant comme une kinase indépendante.

Le modèle d'un Brd4 multifonction agissant seul dans la levée de pause est aussi contredit par l'exemple des gènes hsp70 codant les protéines de choc thermique HSP70. Ces gènes sont classiquement en pause transcriptionnelle dans les cellules; la levée de pause de ces gènes sera induite par un choc thermique. II a récemment été montré que l'inhibition de Brd4 n'affecte pas l'induction des gènes hsp70 en réponse à un choc thermique, alors qu'elle bloque le remodelage de régions spécifiques de la chromatine réalisé par Brd4 [16]. II existe probablement différents schémas de levée de pause selon les gènes, en lien notamment avec le niveau d'accessibilité des régions de la chromatine et des gènes transcrits.

Il est important de souligner que les différents modèles de levée de pause peuvent aussi avoir pour origine des approches expérimentales et des modèles cellulaires différents (drosophile, souris, humain). Le Tableau / récapitule les avantages et les biais des principales techniques employées pour étudier le mécanisme de levée de pause. Initialement, les approches de biochimie ont permis de définir les partenaires de P-TEFb. Elles sont maintenant complétées par les expériences de séquençage, qui permettent d'étudier la production d'ARN naissants, avec la position de régulateurs de la transcription ou de la polymérase sur les gènes. La microscopie de fluorescence permet quant à elle de localiser, dans des cellules analysées individuellement, la formation de complexes de levée de pause, ou de définir la façon dont ces complexes protéiques explorent I'ADN nucléaire pour trouver leurs séquences cibles [17]. Les approches de microscopie en cellules vivantes sont essen- tielles : elles donnent accès à la dimension dynamique qui est primordiale dans les mécanismes de levée de pause. Un des enjeux futurs sera de réussir à intégrer des données multiparamétriques (incluant les big data issues des séquençages à haut débit) en utilisant des outils de modélisation appropriés.

Les différents modèles de recrutement et d'activité de P-TEFb et Brd4 sur les promoteurs, parfois contradictoires, représentent donc probablement chacun une facette différente d'une même histoire (ou d'histoires proches) analysée(s) par différents outils.

\section{Les fonctions du complexe de levée de pause : anticipation, organisation et au-delà}

Outre la possibilité de réaliser une ultime vérification avant que l'élongation transcriptionnelle ne soit engagée, le système de levée de pause présente une rapidité de réaction intéressante. En effet, l'existence de transcrits préchargés avec la polymérase permet une réactivité importante au niveau de la transcription. Cela peut s'avérer utile notamment en conditions de réponse et d'adaptation à un stress cellulaire. Cela permet également d'anticiper et de synchroniser l'activité de gènes régulés de manière périodique, comme suggéré au cours du cycle circadien [18]. La levée de pause confère ainsi, en un certain sens, un art de l'anticipation aux cellules.

Le mécanisme de levée de pause a également été étudié dans le cadre des reprises de transcription après la mitose [19]. Les protéines impliquées dans la levée de pause sont importantes pour le marquage épigénétique, au cours de la mitose, afin d'accélérer la reprise transcriptionnelle, comme cela a été montré 


\begin{tabular}{|c|c|c|}
\hline Technique & Avantages & Inconvénients \\
\hline $\begin{array}{l}\text { Biochimie (élution de fractions, } \\
\text { co-immunoprécipitation) }\end{array}$ & $\begin{array}{l}\text { Détermination des complexes supramoléculaires } \\
\text { Puissance statistique (grand nombre d'événements) }\end{array}$ & $\begin{array}{l}\text { Détection d'interactions indirectes } \\
\text { Moyennage des comportements }\end{array}$ \\
\hline $\begin{array}{l}\text { Microscopie de fluorescence } \\
\text { (FRAP, sptPALM) }\end{array}$ & $\begin{array}{l}\text { Mesures en cellules vivantes de la diffusion des } \\
\text { protéines } \\
\text { Suivi de particules uniques en super-résolution }\end{array}$ & $\begin{array}{l}\text { Besoin de protéines et/ou de locus ectopiques } \\
\text { Coûteux en temps, besoin d'un grand nombre de } \\
\text { mesures pour avoir une puissance statistique } \\
\text { satisfaisante }\end{array}$ \\
\hline $\begin{array}{l}\text { Microscopie de fluorescence } \\
\text { (FRET, FICS) }\end{array}$ & $\begin{array}{l}\text { Mesures et cartographie en cellules vivantes des } \\
\text { interactions entre protéines }\end{array}$ & $\begin{array}{l}\text { Besoin de protéines ectopiques } \\
\text { Coûteux en temps, besoin d'un grand nombre de } \\
\text { mesures pour avoir une puissance statistique } \\
\text { satisfaisante }\end{array}$ \\
\hline ChIP-Seq & $\begin{array}{l}\text { Immunoprécipitation de différentes formes de la } \\
\text { polymérase, sur le génome entier }\end{array}$ & Bruit de fond important \\
\hline RNA-Seq & Détection de l'ensemble des ARN & Sensibilité relativement faible \\
\hline GRO-Seq & Mesures des ARN naissants & Systèmes d'induction nécessaires \\
\hline TT-Seq & Très sensible pour détecter les ARN peu stables & $\begin{array}{l}\text { Détection des ARN synthétisés sur une courte } \\
\text { période ( } 5 \text { minutes) ; pas de détection de trans- } \\
\text { crits « en pause» }\end{array}$ \\
\hline NET-Seq & $\begin{array}{l}\text { Détection des zones de pause de la polymérase, de } \\
\text { formes d'épissage }\end{array}$ & $\begin{array}{l}\text { Non détection des ARN de taille < } 35 \text { nucléotides; } \\
\text { possible poursuite d'élongation lors du protocole; } \\
\text { dégradation possible des ARN }\end{array}$ \\
\hline
\end{tabular}

Tableau I. Techniques utilisées pour étudier le mécanisme de levée de pause transcriptionnelle. FRAP : fluorescence recovery after photobleaching ; FRET : Förster resonance energy transfer; FICS : FRET image correlation spectroscopy; sptPALM : single particle tracking photo-activated localization microscopy ; ChIP-Seq : chromatin immunoprecipitation sequencing; RNA-Seq : ribonucleic acid sequencing; GRO-Seq : global run on sequencing ; TT-Seq : transient transcriptome sequencing ; NET-Seq : native elongating transcript sequencing.

pour Brd4 [20]. Ce marquage épigénétique permet aux cellules de conserver leur profil d'expression, et ainsi leur identité, lors des divisions cellulaires. Il est donc crucial au cours de l'embryogenèse pour assurer l'expression appropriée des gènes du développement dans une période où les divisions cellulaires s'enchaînent rapidement et où les cellules se différencient progressivement.

II ne faut pas, non plus, oublier que P-TEFb module également le recrutement du spliceosome ${ }^{3}$ [53] $(\rightarrow)$ et d'autres étapes ultérieures de la transcription, comme la terminaison et l'export des ARNm (pour revue, voir [21]).

\section{Une chorégraphie complexe et ses défauts d'orchestration}

Comme nous l'avons vu, la levée de la pause transcriptionnelle constitue un mécanisme impliquant la coordination précise de plusieurs partenaires. Des perturbations physiopathologiques peuvent survenir dans cette orchestration et provoquer différents dysfonc-

${ }^{3}$ Le spliceosome est un complexe de ribonucléoprotéines localisé dans le noyau des cellules, dont la fonction est d'assurer la maturation des ARN pré-messager. tionnements. Il est important de noter ici que, bien que les acteurs impliqués possèdent des activités clairement associées à la levée de pause, ils interviennent aussi dans des activités connexes dans le processus de transcription. On ne peut donc exclure que ces activités connexes contribuent aux altérations physiologiques liées aux défauts d'expression ou d'activité de ces acteurs.

Les modèles murins pour étudier la physiopathologie associée au complexe de levée de pause

Bien qu'une certaine redondance puisse autoriser une compensation en cas de défaillance transitoire d'un intervenant du complexe de levée de pause, l'importance physiologique de chacun a été illustrée par les tentatives pour obtenir des modèles transgéniques murins déficients.

À notre connaissance, aucune étude n'a rapporté de souris invalidées pour CTI ou $c d k 9$, et ce n'est que récemment que des modèles murins mimant une telle invalidation ont été décrits. Un modèle de souris 
knock-down (extinction génique) pour CTl a été généré par la technique de gene trap ${ }^{4}$ [22]. Dans ce modèle, les premiers exons de CTI sont maintenus et une expression résiduelle de CTl pleine longueur est observée. Pour cdk9, aucune donnée publiée sur des souris invalidées génétiquement n'est disponible et l'utilisation d'un inhibiteur, le flavopiridol, provoque un arrêt du développement du zygote avant même la formation du blastocyste [23]. II est probable que CTl et cdk9 soient nécessaires pour la survie des cellules souches et des zygotes, d'où l'impossibilité de générer des modèles transgéniques déficients. La suppression de Brd4 est corrélée à une létalité très précoce observée chez les embryons de souris invalidées (knock-out) homozygotes, et les souris hétérozygotes présentent une surmortalité et des défauts majeurs [24]. Brd4 est important pour l'auto-renouvellement des cellules souches embryonnaires, en favorisant la transcription de gènes de pluripotence comme OCT4 (octamer-binding transcription factor 4) via une action sur leurs super enhancers, où elle peut recruter cdk9 [25]. L'invalidation de Clpl (cardiogenic lineage protein 1), I'homogue murin d'HEXIMI, est mieux tolérée aux premiers stades de l'embryogenèse, mais aboutit à une mortalité embryonnaire dès $\varepsilon 16.5$ (16,5 jours de vie embryonnaire), associée à un phénotype d'hypertrophie cardiaque [26].

Dans l'ensemble, les défauts d'expression de chaque acteur du complexe de levée de pause perturbent donc fortement le développement embryonnaire à un stade plus ou moins précoce, même si on ne peut pas formellement relier ces perturbations au mécanisme de levée de pause à proprement parler.

\section{L'importance particulière du complexe de levée de pause pour certains tissus}

Bien que les protéines impliquées dans la levée de pause soient exprimées de manière ubiquitaire dans l'organisme, certains tissus, comme le cœur, semblent plus sensibles aux perturbations de leur activité. Une suractivation de cdk9 a ainsi été observée dans des modèles d'hypertrophie cardiaque, en lien avec une dissociation entre HEXIMI et P-TEFb [27]. La surexpression de cdk9 par l'injection d'ARN chez la souris conduit également à une hypertrophie cardiaque [28]. Des travaux plus récents suggèrent aussi l'implication de Brd4 dans la réponse des cardiomyocytes aux stimulus de stress via une action au niveau des super enhancers associés aux gènes cardiaques dérégulés [29]. Cette activation du complexe P-TEFb et de Brd4 dans ce contexte pathologique a conduit à proposer leur inhibition en thérapie contre I'hypertrophie cardiaque. J $\rho 1$, un inhibiteur de la famille de protéines $B \varepsilon T$, s'est révélé très efficace pour bloquer cette hypertrophie, in vitro et in vivo, en empêchant la surexpression de gènes associés au développement de ce type de pathologie [30].

Le cœur n'est pas le seul tissu particulièrement sensible aux perturbations du complexe de levée de pause. Le système immunitaire l'est également, comme l'indique l'importance de P-TEFb pour l'activité

\footnotetext{
${ }^{4}$ Le principe des stratégies de piégeage (trap) repose sur l'introduction dans le génome d'un gène rapporteur dépourvu d'une partie ou de la totalité de ses éléments de contrôle transcriptionnel. L'expression du gène rapporteur dépendra donc des éléments de régulation agissant en cis de l'unité de transcription, à l'intérieur ou à proximité de laquelle l'insertion aura eu lieu, et sera donc le reflet fidèle de son expression.
}

dans le thymus d'AIRE (auto-immune regulator), un régulateur transcriptionnel clé pour la mise en place d'une tolérance immunitaire [22]. Brd4 est aussi impliqué dans ce processus et un autre inhibiteur de la famille de protéines $B E T, I-B E T 151$, induit une répression des gènes activés par AIRE [31].

Citons un dernier exemple de l'importance des protéines du complexe de levée de pause dans des tissus particuliers, celui de P-TEFb dans le programme d'hypertrophie des mégacaryocytes, cellules donnant naissance aux plaquettes sanguines [32].

\section{Le piratage du système par les virus}

Les virus sont experts dans le détournement à leur profit des machineries des cellules qu'ils infectent. Le VIH (virus de l'immunodéficience humaine) interagit ainsi avec $\mathrm{P}-\mathrm{TEFb}$ et stimule son expression, lors du développement du SIDA (syndrome d'immunodéficience acquise). Plus précisément la protéine virale Tat (transactivator of transcription) recrute $\mathrm{P}$-TEFb pour son activité transcriptionnelle [33], en entrant en compétition avec HEXIMI [34]. Brd4 régule ce mécanisme, et sa surexpression, ou l'expression d'un peptide correspondant au domaine d'interaction de Brd4 avec P-TEFb, empêche la transcription dépendant de Tat [35]. Des inhibiteurs de protéines de la famille BET favorisent au contraire la réactivation et la sortie de latence du virus. Ils ont été proposés comme solution afin d'éradiquer efficacement le virus en faisant sortir les virus latents de leur « cachette » [36].

Brd4 interagit avec des protéines d'autres virus. II favorise notamment la réplication du HPV (human papillomavirus) de type 16 [37]. P-TEFb et Brd4 sont aussi requis pour l'expression par l'EBV (Epstein-Barr virus) de gènes permettant l'immortalisation des cellules qu'il infecte [38]. P-TEFb est également important pour de nombreux autres virus comme le cytomégalovirus ou le virus de l'herpès (pour revue, voir [39]).

\section{Les acteurs de la levée de pause dans les cancers et} leur ciblage

La modulation de l'activité des protéines du complexe de levée de pause est associée au développement de plusieurs types de cancers. Alors qu'il a été proposé, dans un premier temps, que le proto-oncogène $c-M y c$ recrutait I'ARN pol II sur ses gènes cibles et stimulait ainsi l'initiation de leur transcription, il a été montré depuis qu'il agissait vraisemblablement sur l'étape de la levée de pause [40]. L'activité de c-Myc est contrôlée par Brd4, et l'inhibiteur JQl s'est révélé efficace dans des modèles de leucémies, avec un effet dépendant partiellement de la réduction d'expression du proto- 


\begin{tabular}{|c|c|c|c|c|}
\hline $\begin{array}{c}\text { Nom } \\
\text { de l'inhibiteur }\end{array}$ & Cibles & $\begin{array}{l}\text { Phase } \\
\text { d'essai } \\
\text { clinique }\end{array}$ & Résultats & Principaux effets indésirables \\
\hline flavopiridol & $\begin{array}{l}\text { cdk9 et autres } \\
\text { cdk }\end{array}$ & | et I| & Réponses partielles et complètes & $\begin{array}{l}\text { Thrombocytopénie, } \\
\text { neutropénie }\end{array}$ \\
\hline dinaciclib & $\begin{array}{l}\text { cdk9 et autres } \\
\text { cdk }\end{array}$ & | et II & Pas de réponse complète & $\begin{array}{l}\text { Neutropénie, } \\
\text { problèmes gastro-intestinaux }\end{array}$ \\
\hline seliciclib & $\begin{array}{l}\text { cdk9 et autres } \\
\text { cdk }\end{array}$ & I & $\begin{array}{l}\text { Pas de réponse complète, stabilisation } \\
\text { de la maladie }\end{array}$ & Nausées, hypokaliémie réversible \\
\hline OTX015 & famille BET & I & Réponses partielles et complètes & $\begin{array}{l}\text { Thrombocytopénie, } \\
\text { problèmes gastro-intestinaux }\end{array}$ \\
\hline GSK525762 & famille BET & I & $\begin{array}{l}\text { Réponses partielles et stabilisation de } \\
\text { la maladie }\end{array}$ & $\begin{array}{l}\text { Thrombocytopénie, } \\
\text { nausées }\end{array}$ \\
\hline CPI-0610 & famille BET & I & $\begin{array}{l}\text { Réponses complètes et } \\
\text { stabilisation de la maladie }\end{array}$ & Thrombocytopénie \\
\hline BAY 1238097 & famille BET & । & Non rapportés, essai interrompu & Migraines sévères \\
\hline
\end{tabular}

Tableau II. Synthèse des essais cliniques ciblant P-TEFb et Brd4.

oncogène [41]. Une autre preuve du rôle de Brd4 dans la tumorigenèse est apparue à la suite de la découverte de formes de fusion entre Brd4 et NUT (nuclear protein in testis) dans la majorité des carcinomes de la ligne médiane ${ }^{5}$. Ces protéines de fusion assurent un remodelage de la chromatine et de la transcription nécessitant leur phosphorylation par cdk9 [42]. D'autres protéines de fusion responsables de transformation cellulaire et retrouvées dans environ $5 \%$ des leucémies aiguës, aboutissent à la formation de complexes impliquant P-TEFb, induisant une transcription aberrante [43].

Ces études ont encouragé les cliniciens à évaluer l'efficacité d'inhibiteurs de P-T\&Fb et de Brd4 sur des patients lors d'essais cliniques de phase I. Dans le cadre de tumeurs malignes à lymphocytes B, le flavopiridol, inhibiteur de $\operatorname{cdk} 9$, a induit plusieurs rémissions partielles ou complètes, en combinaison avec le bortezomib, un inhibiteur du protéasome [44]. Un des patients a notamment connu une rémission complète après avoir, précédemment, progressé sous traitement par le bortezomib seul. Une phase II a révélé $70 \%$ de rémissions complètes pour des patients atteints de leucémies myéloïdes aiguës et traités par une combinaison d'agents incluant le flavopiridol [45]. Ces résultats encourageants demandent maintenant à être confirmés dans les phases suivantes d'essais cliniques pour préciser leur efficacité.

En ce qui concerne Brd4, un analogue du JQ1, I'OTX015/MK-8628, a induit la régression tumorale chez deux patients atteints de carcinomes de la ligne médiane, sur un groupe de 4 patients [46]. Une autre étude de phase I du même inhibiteur a cependant montré une certaine toxicité chez des patients atteints de myélomes et de lymphomes [47].

${ }^{5}$ Entité constituée de carcinomes peu différenciés centrés sur la ligne médiane (au niveau cervical, médiastinal ou rétropéritonéal).
Le Tableau // récapitule les informations sur les principaux résultats publiés de ces essais cliniques, qui sont détaillés de manière plus exhaustive dans deux revues $[48,49]$.

\section{Les risques de cibler des protéines pléiotropiques}

Le ciblage thérapeutique de P-TEFb et Brd4 a donné lieu à des premiers résultats encourageants dans plusieurs pathologies. Au vu du large spectre d'activités auxquelles participent ces protéines dans l'organisme, une grande prudence reste toutefois de mise.

Les inhibiteurs de Brd4, comme JQl, empêchent la fixation des bromodomaines sur les lysines acétylées, et présentent peu de sélectivité vis-à-vis des protéines appartenant à la famille BET, qui inclut aussi Brd2, Brd3 et Brdt. Ainsi, JQ1 inhibe Brdt, exprimé dans les testicules, et bloque la spermatogenèse chez les souris mâles [50]. Même en considérant la possibilité d'améliorer la spécificité des inhibiteurs pour Brd4, son activité au niveau de gènes impliqués dans la physiologie cardiaque [30] ou participant au renouvellement cellulaire [25], incite donc à la prudence. Une extinction prolongée de Brd4 par des ARN interférents inductibles chez la souris a d'ailleurs mis en évidence des effets délétères sur plusieurs tissus (déplétion de cellules souches intestinales, hyperplasie épidermique et alopécie) [51]. Chez l'homme, les premières données cliniques indiquent une toxicité à court terme de ces inhibiteurs, marquée notamment par des problèmes 
gastro-intestinaux et une thrombocytopénie importante [47]. En ce qui concerne les inhibiteurs de P-TEFb, la tolérance des patients au flavopiridol semble meilleure, avec toutefois une certaine toxicité hématologique, incluant une leucopénie et une thrombocytopénie [44]. Le rôle important de P-TEFb dans la différenciation des mégacaryocytes [32], et la thrombocytopénie marquée que l'on observe en réponse à ces deux types de traitements, laissent suggérer que la formation des plaquettes est particulièrement impactée par ces thérapies.

L'inhibition de Brd4 a été proposée comme stratégie pour éradiquer le VIH, le faisant sortir de sa latence [36]. Néanmoins, la réactivation de virus latents, dans le contexte de traitements chroniques, pourrait poser un problème immunitaire majeur. Brd4 intervient aussi dans la tolérance immunitaire et la sélection positive de lymphocytes $T$ régulateurs, ce qui pourrait contribuer à l'action anticancéreuse que présentent certains inhibiteurs de Brd4 [31]. À l'inverse, la famille de protéines BET participe à la différenciation de certains lymphocytes $T$ auxiliaires et l'inhibiteur J $\mathrm{l} 1$ permet de traiter des maladies auto-immunes dans des modèles murins [52]. L'impact sur le système immunitaire de tels inhibiteurs représente donc un autre aspect à considérer en thérapie à long terme.

Il est encore trop tôt pour appréhender les effets à moyen terme des inhibiteurs de protéines la famille BET, mais les éléments que nous avons abordés incitent à la prudence, notamment lors de traitements prolongés.

\section{Conclusion et perspectives}

La levée de pause constitue un mécanisme de contrôle de la transcription permettant une réponse transcriptionnelle rapide et synchronisée. Différents modèles reposant sur des acteurs clés, P-TEFb et Brd4, ont été proposés, correspondant sans doute à différentes facettes d'une réalité dynamique. L'évolution d'outils a permis et permet de raffiner constamment les connaissances à ce sujet. Le développement du séquençage à haut débit sur cellules individuelles apporte ainsi une vision plus nette de la régulation au niveau de différents promoteurs. De même, l'amélioration des techniques de microscopie et de biophotonique nous permet d'obtenir de plus en plus d'informations quantitatives sur les complexes moléculaires qui participent au processus. Le développement d'approches biophysiques, pour analyser les dynamiques moléculaires, constitue un autre atout pour comprendre des agencements variés qui interviennent dans une même cellule vivante, et pour permettre une étude spatiale et dynamique de cette régulation transcriptionnelle. Un des défis qui reste à relever sera de réussir à croiser les données issues des approches multi-omics (épigénomique, transcriptomique, etc.) et celles de la microscopie autour d'une stratégie de recherche commune.

Les processus d'initiation, de levée de pause, d'élongation, d'épissage et de terminaison de la transcription sont intriqués et présentent des acteurs communs. Il est ainsi difficile d'attribuer, de manière simpliste, une action propre à chaque musicien sur la partition. Un défi futur sera donc d'adapter nos études afin d'examiner et comprendre ce véritable travail d'orchestre, plutôt que la somme des prestations de différents solistes. En ce sens, la biologie des systèmes peut être d'un grand intérêt pour appréhender de tels systèmes non linéaires, fondés sur des interactions croisées et des boucles de rétrocontrôle.

Enfin, la recherche translationnelle représente un enjeu majeur de la recherche autour du complexe de levée de pause. Les acteurs moléculaires qui y participent, constituent en effet des cibles prometteuses pour plusieurs pathologies. Toutefois, de par l'action ubiquitaire des molécules susceptibles d'être ciblées, notamment les protéines de la famille BET (qui sont impliquées dans la physiologie cardiaque, la tolérance immunitaire, le renouvellement intestinal, la spermatogenèse, la latence de virus, etc.), il sera important d'évaluer la toxicité et l'impact des agents thérapeutiques utilisés lors de traitements prolongés. $\diamond$

\section{SUMMARY}

P-TEFb and Brd4: actors of the transcription pause release as therapeutical targets

Most cell physiology events are dictated by the integration of perceived signals and the elaboration by cells of adapted answers via the execution of proper transcriptional programs. In order to ensure an optimal control of these answers, many regulation mechanisms have been selected throughout the evolution, thus allowing to fine-tune transcript expression. The transcriptional pause and its release by P-TEFb (Positive Transcription Elongation Factor) have been evidenced two decades ago. Since then, the importance of such mechanisms has been highlighted by the association between alterations of this machinery and the appearance of diseases. P-TEFb and Brd4 have thus recently emerged as potential therapeutical targets for cancers and AIDS notably. In this review, we present a brief case history and an upto-date synthesis of models for transcriptional pause release. We later discuss on the pathophysiological processes associated with this mechanism and clinical trials targeting Brd4 and P-TEFb. $\diamond$

\section{LIENS D'INTÉRÊT}

Les auteurs déclarent n'avoir aucun lien d'intérêt concernant les données publiées dans cet article.

\section{RÉFÉRENCES}

1. Day DS, Zhang B, Stevens SM, et al. Comprehensive analysis of promoterproximal RNA polymerase II pausing across mammalian cell types. Genome Biol 2016; $17: 120$.

2. Jonkers I, Lis JT. Getting up to speed with transcription elongation by RNA polymerase II. Nat Rev Mol Cell Biol 2015 ; 16 : 167-77.

3. Marshall NF, Price DH. Purification of P-TEFb, a transcription factor required for the transition into productive elongation. J Biol Chem $1995 ; 270$ : 12335-8. 


\section{RÉFÉRENCES}

4. Cho S, Schroeder S, Ott M. Cycling through transcription : posttranslational modifications of P-TEFb regulate transcription elongation. Cell Cycle Georget Tex 2010 ; 9 : 1697-705.

5. Muniz L, Kiss T, Egloff S. Perturbations de la transcription liées à une dérégulation de P-TEFb : cancer, Sida et hypertrophie cardiaque. Med Sci (Paris) $2012 ; 28: 200-5$

6. Nguyen VT, Kiss T, Michels AA, et al. 7SK small nuclear RNA binds to and inhibits the activity of CDK9/cyclin T complexes. Nature $2001 ; 414: 322-5$

7. Kobbi L, Demey-Thomas $\varepsilon$, Braye F, et al. An evolutionary conserved Heximl peptide binds to the Cdk9 catalytic site to inhibit P-TEFb. Proc Natl Acad Sci USA 2016 ; 113 : 12721-6.

8. Quaresma AJ, Bugai A, Barboric M. Cracking the control of RNA polymerase II elongation by 7SK snRNP and P-TEFb. Nucleic Acids Res $2016 ; 44$ : 7527-39.

9. Jang MK, Mochizuki K, Zhou M, et al. The bromodomain protein Brd4 is a positive regulatory component of P-TEFb and stimulates RNA polymerase II-dependent transcription. Mol Cell 2005 ; $19: 523-34$.

10. McNamara RP, Reeder JE, McMillan $E A$, et al. KAPl recruitment of the 7SK snRNP complex to promoters enables transcription elongation by RNA polymerase II. Mol Cell $2016 ; 61: 39-53$.

11. Bidaux G, Le Nézet C, Pisfil MG, et al. FRET image correlation spectroscopy reveals mapiiindependent P-TEFb recruitment on chromatin. Biophys / 2018 ; 114 : 522-33.

12. Liu W, Ma $Q$, Wong $\mathrm{K}$, et al. Brd4 and JMJD6-associated anti-pause enhancers in regulation of transcriptional pause release. Cell $2013 ; 155: 1581-95$.

13. Devaiah BN, Case-Borden C, Gegonne A, et al. BRD4 is a histone acetyltransferase that evicts nucleosomes from chromatin. Nat Struct Mol Biol $2016 ; 23: 540-8$.

14. Baranello L, Wojtowicz D, Cui K, et al. RNA polymerase II regulates topoisomerase 1 activity to favor efficient transcription. Cell $2016 ; 165: 357-71$.

15. Devaiah BN, Singer DS. Cross-talk among RNA polymerase II kinases modulates C-terminal domain phosphorylation. J Biol Chem 2012 ; 287 : 38755-66.

16. Col $\varepsilon$, Hoghoughi N, Dufour $S$, et al. Bromodomain factors of BET family are new essential actors of pericentric heterochromatin transcriptional activation in response to heat shock. Sci Rep $2017 ; 7: 5418$.

17. Izeddin I, Récamier V, Bosanac L, et al. Single-molecule tracking in live cells reveals distinct target-search strategies of transcription factors in the nucleus. elife $2014 ; 3$.

18. Westermark PO. Linking core promoter classes to circadian transcription. PLoS Genet 2016 ; 12 : el006231.

19. Yang Z, He N, Zhou Q. Brd4 recruits P-TEFb to chromosomes at late mitosis to promote Gl gene expression and cell cycle progression. Mol Cell Biol $2008 ; 28: 967-76$.

20. Zhao R, Nakamura T, Fu Y, et al. Gene bookmarking accelerates the kinetics of post-mitotic transcriptional re-activation. Nat Cell Biol $2011 ; 13: 1295-304$.

21. Brès V, Yoh SM, Jones KA. The multi-tasking P-TEFb complex. Curr Opin Cell Biol $2008 ; 20$ : 334-40.

22. Oven I, Brdicková $\mathrm{N}$, Kohoutek J, et al. AIRE recruits $\mathrm{P}$-TEFb for transcriptional elongation of target genes in medullary thymic epithelial cells. Mol Cell Biol $2007 ; 27: 8815-23$.

23. Oqani RK, Lin T, Lee JE, et al. Inhibition of P-TEFb disrupts global transcription, oocyte maturation, and embryo development in the mouse. Genes $2016 ; 54: 470-82$.

24. Houzelstein D, Bullock SL, Lynch DE, et al. Growth and early postimplantation defects in mice deficient for the bromodomain-containing protein Brd4. Mol Cell Biol $2002 ; 22$ : 3794-802.

25. Di Micco R, Fontanals-Cirera B, Low V, et al. Control of embryonic stem cell identity by BRD4dependent transcriptional elongation of super-enhancer-associated pluripotency genes. Cell Rep $2014 ; 9: 234-47$

26. Huang F, Wagner M, Siddiqui MA. Ablation of the CLP-1 gene leads to down-regulation of the HANDl gene and abnormality of the left ventricle of the heart and fetal death. Mech Dev 2004 ; $121: 559-72$

27. Espinoza-Derout J, Wagner M, Shahmiri K, et al. Pivotal role of cardiac lineage protein-1 (CLP-1) in transcriptional elongation factor $\mathrm{P}-\mathrm{TEFb}$ complex formation in cardiac hypertrophy. Cardiovasc Res $2007 ; 75: 129-38$.

28. Wagner KD, Wagner $\mathrm{N}$, Ghanbarian $\mathrm{H}$, et al. RNA induction and inheritance of epigenetic cardiac hypertrophy in the mouse. Dev Cell $2008 ; 14: 962-9$.

29. Stratton MS, Lin CY, Anand P, et al. Signal-dependent recruitment of BRD4 to cardiomyocyte super-enhancers is suppressed by a microRNA. Cell Rep 2016; 16:1366-78.

30. Anand P, Brown JD, Lin CY, et al. BET bromodomains mediate transcriptional pause release in heart failure. Cell $2013 ; 154: 569-82$.

31. Yoshida H, Bansal K, Schaefer U, et al. Brd4 bridges the transcriptional regulators, Aire and P-TEFb, to promote elongation of peripheral-tissue antigen transcripts in thymic stromal cells. Proc Natl Acad Sci USA $2015 ; 112$ : ع4448-57.
32. Elagib KE, Rubinstein JD, Delehanty LL, et al. Calpain 2 activation of P-TEFb drives megakaryocyte morphogenesis and is disrupted by leukemogenic GATAl mutation. Dev Cell $2013 ; 27: 607-20$.

33. Mancebo HS, Lee G, Flygare J, et al. P-TEFb kinase is required for HIV Tat transcriptional activation in vivo and in vitro. Genes Dev $1997 ; 11: 2633$ 44.

34. Barboric M, Yik JHN, Czudnochowski N, et al. Tat competes with HEXIMl to increase the active pool of P-TEFb for HIV-1 transcription. Nucleic Acids Res $2007 ; 35: 2003-12$.

35. Bisgrove DA, Mahmoudi T, Henklein P, et al. Conserved P-TEFb-interacting domain of BRD4 inhibits HIV transcription. Proc Natl Acad Sci USA 2007 ; $104: 13690-5$.

36. Lu P, Qu X, Shen Y, et al. The BET inhibitor OTX015 reactivates latent HIV-1 through P-TEFb. Sci Rep $2016 ; 6: 24100$.

37. Wang X, Helfer CM, Pancholi N, et al. Recruitment of Brd4 to the human papillomavirus type 16 DNA replication complex is essential for replication of viral DNA. J Virol $2013 ; 87: 3871-84$.

38. Palermo RD, Webb HM, West MJ. RNA polymerase II stalling promotes nucleosome occlusion and $\mathrm{pTEFb}$ recruitment to drive immortalization by Epstein-Barr virus. PLoS Pathog $2011 ; 7$ : e1002334.

39. Zaborowska J, Isa NF, Murphy S. P-TEFb goes viral. BioEssays News Rev Mol Cell Dev Biol 2016 ; 38 (suppl 1) : S75-85.

40. Rahl PB, Lin Cy, Seila AC, et al. c-Myc regulates transcriptional pause release. Cell $2010 ; 141: 432-45$.

41. Zuber J, Shi J, Wang $\varepsilon$, et al. RNAi screen identifies Brd4 as a therapeutic target in acute myeloid leukaemia. Nature $2011 ; 478$ : 524-8.

42. Wang R, Cao X-J, Kulej K, et al. Uncovering BRD4 hyperphosphorylation associated with cellular transformation in NUT midline carcinoma. Proc Natl Acad Sci USA 2017 ; 114 : ع5352-61.

43. Yokoyama A, Lin M, Naresh A, et al. A higher-order complex containing AF4 and ENL family proteins with P-TEFb facilitates oncogenic and physiologic MLL-dependent transcription. Cancer Cell $2010 ; 17$ : 198-212.

44. Holkova B, Kmieciak M, Perkins $\varepsilon B$, et al. Phase I trial of bortezomib (PS341 ; NSC 681239) and nonhybrid (bolus) infusion schedule of alvocidib (flavopiridol; NSC 649890) in patients with recurrent or refractory indolent B-cell neoplasms. Clin Cancer Res $2014 ; 20$ : 5652-62.

45. Zeidner JF, Foster MC, Blackford AL, et al. Randomized multicenter phase II study of flavopiridol (alvocidib), cytarabine, and mitoxantrone (FLAM) versus cytarabine/daunorubicin $(7+3)$ in newly diagnosed acute myeloid leukemia. Haematologica $2015 ; 100: 1172-9$.

46. Stathis A, Zucca $\varepsilon$, Bekradda M, et al. Clinical response of carcinomas harboring the BRD4-NUT oncoprotein to the targeted bromodomain inhibitor OTX015/MK-8628. Cancer Discov 2016; $6: 492-500$.

47. Amorim S, Stathis A, Gleeson M, et al. Bromodomain inhibitor OTX015 in patients with lymphoma or multiple myeloma : a dose-escalation, openlabel, pharmacokinetic, phase 1 study. Lancet Haematol 2016; 3 : e196-204.

48. Morales F, Giordano A. Overview of CDK9 as a target in cancer research. Cell Cycle Georget Tex 2016; 15 : 519-27.

49. Stathis A, Bertoni F. BET Proteins as targets for anticancer treatment. Cancer Discov 2018 ; 8: 24-36.

50. Matzuk MM, McKeown MR, Filippakopoulos P, et al. Small-molecule inhibition of BRDT for male contraception. Cell 2012 ; $150: 673-84$

51. Bolden JE, Tasdemir N, Dow $L E$, et al. Inducible in vivo silencing of Brd4 identifies potential toxicities of sustained BET protein inhibition. Cell Rep 2014 ; 8 : 191929.

52. Mele DA, Salmeron A, Ghosh S, et al. BET bromodomain inhibition suppresses TH17-mediated pathology. J Exp Med 2013 ; 210 : 2181-90.

53. Dujardin G, Daguenet $\varepsilon$, Bernard DG, et al. L'épissage des ARN pré-messagers : quand le splicéosome perd pied. Med Sci (Paris) 2016 ; 32 : 1103-10.

\section{TIRÉS À PART}

A. Furlan

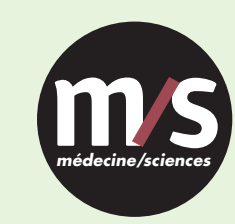

Tarifs d'abonnement $\mathrm{m} / \mathrm{s}-2018$

Abonnez-vous

à médecine/sciences
$>$ Grâce à $\mathrm{m} / \mathrm{s}$, vivez en direct les progrès des sciences biologiques et médicales

Bulletin d'abonnement page 754 dans ce numéro de $\mathrm{m} / \mathrm{s}$

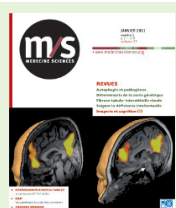

